\title{
Impregnation of Composite Materials: a Numerical Study
}

\author{
Elliott Baché ${ }^{1,2}$ (D) . Chloé Dupleix-Couderc ${ }^{1}$. \\ Eric Arquis $^{2}$ - Isabelle Berdoyes ${ }^{1}$
}

Received: 12 October 2017 / Accepted: 20 November 2017 / Published online: 19 December 2017

(C) The Author(s) 2017. This article is an open access publication

\begin{abstract}
Oxide ceramic matrix composites are currently being developed for aerospace applications such as the exhaust, where the parts are subject to moderately high temperatures $\left(\approx 700{ }^{\circ} \mathrm{C}\right)$ and oxidation. These composite materials are normally formed by, among other steps, impregnating a ceramic fabric with a slurry of ceramic particles. This impregnation process can be complex, with voids possibly forming in the fabric depending on the process parameters and material properties. Unwanted voids or macroporosity within the fabric can decrease the mechanical properties of the parts. In order to design an efficient manufacturing process able to impregnate the fabric well, numerical simulations may be used to design the process as well as the slurry. In this context, a tool is created for modeling different processes. Thétis, which solves the Navier-Stokes-Darcy-Brinkman equation using finite volumes, is expanded to take into account capillary pressures on the mesoscale. This formulation allows for more representativity than for Darcy's law (homogeneous preform) simulations while avoiding the prohibitive simulation times of a full discretization for the composing fibers at the representative elementary volume scale. The resulting tool is first used to investigate the effect of varying the slurry parameters on impregnation evolution. Two different processes, open bath impregnation and wet lay-up, are then studied with emphasis on varying their input parameters (e.g. inlet velocity).
\end{abstract}

Keywords Ceramic matrix composites · Impregnation · Finite volumes · Capillary pressure

Elliott Baché

elliottbache@hotmail.com

1 Institute of Technology Antoine de Saint Exupéry, Campus de Bordeaux-Talence, Esplanade des Arts et Métiers, 33405, Talence Cedex, France

2 I2M - CNRS, Bordeaux INP, Université de Bordeaux, Arts et Métiers ParisTech, 16 avenue Pey-Berland, 33607 Pessac Cedex, France 


\section{Introduction}

Due to environmental and economic concerns, weight reduction in aeronautical structures has lead to the use of composite materials. They were first used for structural parts of planes or engines that were not exposed to high temperatures. Recently, however, with the increase of aeronautical engine temperature, new ceramic matrix composite materials (CMCs) have been developed to replace the traditional metals used in hot parts. Two main materials have been developped for these applications: SiC CMCs and oxide CMCs. SiC CMCs have good mechanical properties and can endure high temperatures (up to $1400{ }^{\circ} \mathrm{C}$ ). They are mainly considered for inner parts of the engine that are subject to large mechanical loads. Oxide $\mathrm{CMCs}$, on the other hand, they do not have quite as favorable mechanical properties as $\mathrm{SiC}$ materials, but they are cheaper to produce. For these reasons they are mainly studied for applications such as the engine exhaust, where the maximum temperature is around $700{ }^{\circ} \mathrm{C}$ and a large resistance to loads is unnecessary. In this context, oxide CMCs are considered in this study.

The process to manufacture oxide CMCs consists of three different steps. The first step is the impregnation of the fabric by a slurry containing the ceramic powder. This can be achieved in different ways such as injection of the slurry or impregnation of the fabric in a bath. The second step is the drying of the impregnated fabric, which consists in removing the liquid constituants of the slurry. Finally, a partial sintering of the material ensures the cohesion of the different constituants. The micro-porosity of the matrix, created during the partial sintering step, is used to deviate the cracks which can appear in the loaded material. For this reason, oxide CMCs have a high level of porosity (about 25-30\%): the material is thus tolerant to damages. On the other hand, macro-porosity, which is unintentionally formed voids, can decrease the mechanical properties of the material.

The process to create oxide CMCs is currently being researched around the world [20, 22], and the optimal process parameters and methods are still an open debate. In particular, the first step of the manufacturing process is a complex subject with many possible final saturation profiles due to the different methods and materials that can be used. For instance, the impregnation is greatly affected by the process parameters (e.g. pulling velocity) and the materials parameters such as slurry viscosity and fabric geometry, with each set of slurry and fabric properties requiring a sufficient pressure and impregnation time [24]. The outcome of the impregnation is very important to the mechanical properties of the final product. During impregnation, one must ensure that the fiber tows are well impregnated with the slurry to control the macro-porosity within the material and ensure a good cohesion between fibers and matrix. Since there are still many methods and materials that must be tested to optimize the oxide CMC manufacturing process, numerical simulations are used in this study to gain knowledge of which materials and methods are most likely to produce better impregnation.

Accurate simulations of the process to obtain material composites can be difficult to achieve due to complex topologies. The fiber tows are generally composed of hundreds to thousands of fibers.

Such a large number of micron-scale individual fibers, coupled with the size of a typical representative elementary volume $(\mathrm{REV})(\approx 1 \mathrm{~cm} \mathrm{x} 1 \mathrm{~cm} \times 0.1 \mathrm{~cm})$ of fabric makes a full discretization nearly impossible. The simulation scale must be increased if the simulation of global impregnation of a fabric is to be feasible. For this reason, simulations are often carried out on the mesoscale [17] or macroscale [15]. The macroscale can be modeled by simplifying to a generalized porous medium and assigning a permeability and porosity 
to the fabric preform $[13,23,26]$. This modelization relies on using the Darcy equation to resolve the flow. Due to this method's simplicity, calculations are normally simple and fast. Of course, a certain degree of accuracy is lost when simplifying to a homogeneous porous medium; the fabrics are actually woven with spacings between the tows and are not homogeneous. In addition, CMCs are typically composed of various superimposed layers, creating more spaces between tows. For this reason, the fabric preforms are not very homogeneous, making simulations more inaccurate in smaller geometries. Another of the main disadvantages of this generalization is that it cannot take into account wetting phenomena since these are highly dependent on the geometry at small scales. These phenomena can be significant depending on the boundary conditions of the process to be modeled. In resin transfer molding (RTM) for example, the forced injection pressures will normally overshadow the capillary forces $[4,28]$ between fiber tows and can perhaps be neglected depending on fiber tow geometry, injection pressures, etc. For many processes however, the capillary pressures are significant within tows and can be significant between tows, and may render a Darcy simplicification inapt [1].

For modeling many different processes, the mesoscale must be treated in order to correctly evaluate the contributions of the pressure gradients and capillary forces. At the mesoscale, the fabric preform can be modeled as being composed of fiber tows. The equations are a bit more complex than for the macroscale, requiring the Navier-Stokes equations in order to solve the non-Darcy behavior between tows as well as an added Darcy term to model the behavior within tows. On the other hand, even though equations are more complex, the physical characteristics of the preforms are much more readily deduced. At this scale, the fiber tows are discretized and are assumed to be homogeneous porous media. This contrasts with the homogenization of the entire preform when treating the problem at the macroscale. The tows, which are essentially arrays of parallel cylinders, are much easier to characterize than the entire preform, which is composed of complex weaves superimposed upon each other. Many studies have previously been conducted to obtain flow characteristics around cylinders, allowing the permeability, porosity, and capillary pressure to be approximated from correlations presented in the literature $[2,18]$. Special care should be taken however when defining the spacing between cylinders, since a change in spacing between fibers will cause a change in porosity, permeability, and capillary pressure.

The mesoscale model presented here provides information on the completeness of the impregnation, taking into account capillary as well as pressure gradient phenomena. This information can be used to design processes that minimize macroporosity, which will maximize mechanical properties in the final product after the remaining drying and sintering processes. In this study, the different properties of the slurry are explored, establishing practical limits and aiding in defining what slurry property values should be sought to obtain a well impregnated fabric. Various processes are explored to determine which ones will result in the most complete impregnation.

This article is organized in the following sections. The first section describes the methodology to create the model for the different processes under consideration. It also describes the parameters used in the study and how they are obtained. The second section describes a simple validation of the solver for capillary rise in a permeable fiber tow and the results for the different tests. This section presents results for spontaneous impregnation of a quantity of slurry into the fabric, varying the physical properties of the slurry to ascertain which parameters are most important and how their variation affects impregnation. It also explores two different impregnation processes and the parameters associated with them. The third section summarizes the findings and states conclusions. 


\section{Methodology}

\subsection{Industrial Application}

The oxide CMCs studied here are comprised of Nextel 610 fiber fabric ( 8 harness satin weave) and a pure alumina matrix. The alumina matrix is impregnated into the fabric by means of a solvent that evaporates afterwards. Since the solvent is no longer present in the finished product, there is a large degree of freedom in its design. For this reason, the slurry parameters that are studied here are varied through ranges that correspond to standard solvents. The fabric on the other hand is fixed as the Nextel 610.

In addition to varying the material properties, the process parameters are also varied. Two main processes are presented in this article: open bath impregnation (Section 2.6) and wet lay-up (Section 2.7). The open bath impregnation process is a fairly standard process [11] in which the filament is pulled through a resin bath. On the other hand, the conditions of the wet lay-up process can be achieved in many ways, applying resin and pressure to the fabric. It corresponds to many possible processes (e.g. with rollers, spatulas, etc.).

The objective of this study is thus to numerically model spontaneous impregnation to explore the slurry parameters, and the open bath and the wet lay-up processes for different values of the slurry properties in hopes of gaining a deeper understanding of the processes in question and of defining the key parameters of the process.

\subsection{Governing equations}

A mesoscale model must be able to simulate flow between tows as well as within tows, where capillary effects are important [1]. For this reason, in addition to the standard inertial, viscous, gravity, and pressure terms in the Navier-Stokes equations, extra terms are added to account for flow through porous media, capillary pressure, and surface tension. Bond numbers near and lower than unity correspond to problems that require consideration of the surface tension. This assumption gives way to the definition of the capillary length:

$$
l_{c}=\left(\frac{\sigma}{\rho g}\right)^{1 / 2},
$$

where $l_{c}$ is the capillary length, $\rho$ is the density, g is gravity, and $\sigma$ is the surface tension of the fluid. Given our application, the capillary length is approximately $1.5 \mathrm{~mm}$, which is the same scale as the fiber tows, thus validating the use of a surface tension term in the Navier-Stokes (-Darcy-Brinkman) equation, especially within the tows. Whereas within the tows a capillary pressure term models the effect of the wetting interaction between fluid and solid, wetting effects are assumed to have little effect outside of the tows. The assumption is made that between tows the viscous forces and the pressure gradients resulting from the boundary conditions will be dominant [1]. Aside capillary effects, the porous media present in the domain requires the Navier-Stokes equation to be modified to become the NavierStokes-Darcy-Brinkman equation $[9,10,14]$ with added terms for the capillary pressure and surface tension. The final one-fluid incompressible equation set used in Thétis is:

$$
\begin{gathered}
\nabla \cdot \mathbf{u}=0 \\
\rho\left(\frac{\partial \mathbf{u}}{\partial t}+\frac{1}{\epsilon} \mathbf{u} \cdot \nabla \mathbf{u}\right)=-\epsilon \nabla p+\rho \epsilon \mathbf{g}+\nabla \cdot\left(\mu\left[\nabla \mathbf{u}+\nabla^{T} \mathbf{u}\right]\right)+\mathbf{F}_{S T}-\frac{\mu \epsilon}{K} \mathbf{u}-\nabla p_{c} \\
\frac{\partial C}{\partial t}+\mathbf{u} \cdot \nabla C=0
\end{gathered}
$$


where $\mathbf{u}$ is the velocity vector, $\mathrm{t}$ is time, $\mathrm{p}$ is pressure, $\mathbf{g}$ is the gravity vector, $\mu$ is viscosity, $\kappa$ is the permeability, $\epsilon$ is porosity, $p_{c}$ is the capillary pressure, and $\mathrm{C}$ is the color function associated with the liquid phase $(C=1$ for grid points inside the slurry, $C=0$ for grid points outside the slurry). The added porous term was first researched by Brinkman [9] for high porosity swarms of particles and was validated with experimental results. Results from Brinkman's equations were compared to experimental results from Carman for the ratio of drag forces on particles to the pressure drop [12] and were found to coincide at high porosities. At the porosity used in this study, 0.487 (see Section 2.3.1), Brinkman's results are also very similar to Carman's. This formulation is thus kept, and a validation with a simple geometry is carried out in Section 2.4.

The surface tension term is expressed using the continuum surface force model [8] and is

$$
\mathbf{F}_{S T}=\sigma \nabla \cdot\left(\frac{\nabla C^{S}}{\left\|\nabla C^{S}\right\|}\right) \nabla C^{S},
$$

where $C^{S}$ is the smoothed color function. For sharp interfaces (as is physically the case at most scales) between gas and liquid, the color function usually varies from 0 to 1 over the width of only one discretization cell. Since the surface tension term relies on second-order derivatives, this term can be error-prone due to a lack information in the adjacent cells to form at least a three-point stencil. For this reason, the color function is smoothed, solving a Helmholtz to equation to artificially diffuse the interface. More details of the method can be found in [19].

The capillary pressure is calculated assuming arrays of parallel cylinders, following the methodology in [31] and can be expressed as

$$
\begin{gathered}
p_{c}=\frac{F}{D_{f}} \frac{1-\epsilon}{\epsilon} \sigma \cos \theta \\
F= \begin{cases}4 & \text { for axial flow } \\
2 & \text { for perpendicular flow }\end{cases}
\end{gathered}
$$

where $D_{f}$ is the diameter of the fibers, and $\theta$ is the static contact angle of the liquid with respect to the surface of the fibers.

The slurry is composed of solvent and particles. Segregation can thus occur during the impregnation phase. Nevertheless, in this study, we have considered as a first step that the slurry is uniform and does not evolve during the entire simulation. Instead, the slurry is assumed to be uniform throughout impregnation. In parallel, work is underway to study the effect of segregation on impregnation in CMCs [5]. The influence of temperature is not taken into account.

\subsection{Governing Equation Parameters}

\subsubsection{Fabric Parameters}

The main parameters that must be approximated for the fabric are the permeability and porosity. These parameters will remain fixed throughout this study and depend solely on the fixed geometry. The fabric geometry may be seen in Fig. 1. Given the geometry of the fabric and the individual fiber tows, the cross-sectional area of the tows may be computed. In the Nextel610 fabric each tow is composed of 400 fibers, and the fibers measure 1012 microns in diameter. Taking an average of 11 microns and using the previously computed 

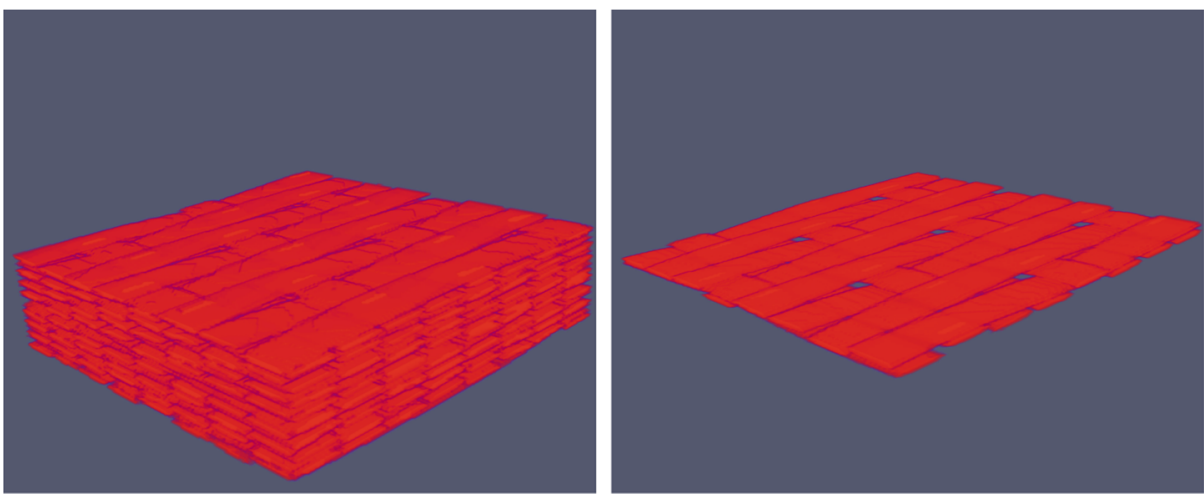

Fig. 1 Left: REV, right: 1 layer of REV

cross-sectional area, a porosity of 0.487 may be deduced. Correlations for cylinders allow calculation of the permeability (7) in the axial and perpendicular directions [27].

$$
\begin{gathered}
\kappa=C\left(\sqrt{\frac{1-\phi_{c}}{1-\phi}}-1\right)^{2.5} R_{f}^{2} \\
C= \begin{cases}16 / 9 \sqrt{2} \pi & \text { for square arrays } \\
16 / 9 \sqrt{6} \pi & \text { for hexagonal arrays }\end{cases}
\end{gathered}
$$

where $\phi_{c}$ is the critical value of porosity below which there is no permeating flow, equal to $1-\pi / 2 \sqrt{3}$ for hexagonal arrays. For this study, a hexagonal array is assumed when calculating maximum and minimum permeability values. Since this study is more concerned with tendencies than absolute resultant saturation, the exact value of the permeability and thus of the constants is not crucial.

\subsubsection{Slurry Parameters}

The slurry parameters, as opposed to the fabric parameters, will be varied in this study. Density, surface tension, wetting contact angle, and viscosity are the slurry parameters that can possibly affect the impregnation results. These parameters are first measured for two different slurries, whose compositions differ from each other. These measurements help decide the range of the parameters according to changes in concentrations and components of the slurry. In this way, the parameter ranges in Table 1 are defined.

The density is measured experimentally with a standard pycnometer. The surface tension of the two slurries is measured using an OCA40 Data Physics goniometer. The viscosity measurements are made with a Brookfield LVDV II+ using a $38 \mathrm{~mm}$ diameter beaker, an

Table 1 Parameter ranges

\begin{tabular}{llll}
\hline Parameter & Units & Minimum & Maximum \\
\hline Density & $\mathrm{kg} / \mathrm{m}^{3}$ & 2092 & 2540 \\
Surface tension & $\mathrm{N} / \mathrm{m}$ & 0.02 & 0.08 \\
Contact angle & $\circ$ & 40 & 80 \\
Viscosity & $\mathrm{Pa} \cdot \mathrm{s}$ & 0.1 & 0.67 \\
\hline
\end{tabular}


LV2 mobile, and 20 RPMs. The sensitivity for these measurements is $0.015 \mathrm{~Pa} \cdot \mathrm{s}$. The values of the viscosities are kept constant throughout all of the simulations.

The wetting contact angle is measured using the Wilhelmy balance technique. The standard Wilhelmy plate balance uses a platinum plate that is dipped in a liquid and slowly extracted until fully outside of the liquid. The force required to withdraw the plate at the moment just before full extraction is the surface tension force. Assuming a contact angle of $0^{\circ}$, the surface tension of the liquid can be derived from Eq. 8 and the physical dimensions of the plate.

$$
F=p_{p} \sigma \operatorname{Cos} \theta
$$

where $p_{p}$ is the perimeter of the plate. This experiment used the $3 \mathrm{~S}$ Surface, Science, Study Wilhelmy tensiometer and a modified protocol to calculate the contact angle. Instead of using the platinum plate, a single, deenzymed Nextel610 fiber tow was used. In this case, the measured surface tension force and the previously measured surface tension coefficient and physical dimensions of the fiber tow allowed calculation of the contact angle. The results are presented in Table 2. The contact angle's range (see Table 1) for the gravity tests in Section 2.5 is centered around this contact angle with a relatively wide range: $40^{\circ} \leq \theta \leq$ $80^{\circ}$.

\subsection{Validation}

Thétis [32] has previously been validated for a wide range of fluid dynamics problems. Among these, various porous medium problems have been treated with Thétis including water flow on beaches [16], reactive flow in composite materials [5], and liquid flow through mushy layers during solidification $[6,29,30]$. The code has also been used to study wetting effects at the microscale [7]. These studies did not however include the capillary pressure term that has been implemented in this study. For this reason, a validation for the revised equations is undertaken. The revised Thétis code is validated for a $2 \mathrm{D}$ permeable fiber tow with a capillary pressure inside the fiber tow. This $2 \mathrm{D}$ permeable fiber tow is meant to be an approximation of the permeable fiber tows that make up the fabric. Here, the individual fibers that comprise the tow are not discretized. Instead, the fiber tow is modeled as an equivalent porous medium. Under ideal conditions, the flow through a permeable fiber tow can be simplified to follow Darcy's law, which states that an applied pressure will result in a constant velocity for flow through a porous medium (see Eq. 9).

$$
Q=-\frac{\kappa A}{\mu} \nabla p,
$$

Table 2 Contact angle measurements between slurry and fiber tow

\begin{tabular}{llll}
\hline Run & Contact angle $\left(^{\circ}\right)$ & Run & Contact angle $\left(^{\circ}\right)$ \\
\hline 1 & 60.4 & 5 & 51.0 \\
2 & 66.2 & 6 & 57.4 \\
3 & 57.4 & 7 & 62.4 \\
4 & 57.4 & 8 & 70.8 \\
Average & 60.3 & & \\
Standard deviation & 6.1 & & \\
\hline
\end{tabular}


where $\mathrm{Q}$ is the flow rate and $\mathrm{A}$ is the cross-sectional area of the fiber tow. Taking into account the porosity and including the capillary pressure, the velocity of the liquid through a permeable fiber tow [3] can be expressed as:

$$
v=-\frac{\kappa}{\mu \epsilon} \frac{p_{c}-\rho g h}{h},
$$

where $\mathrm{h}$ is the height the liquid has risen in the vertical permeable fiber tow. For the validation case, a permeable fiber tow of finite length $(80 \mathrm{~mm} \times 2 \mathrm{~mm})$ is created and given a porosity of 0.487 and a permeability of $1.22 \cdot 10^{-12} \mathrm{~m}^{2}$. The fiber tow is initially dry and has its lower extremity dipped in a very large reservoir (50x its width). The capillary pressure is assumed to be $9000 \mathrm{~Pa}$. The geometry as entered into Thétis may be seen in Fig. 2 . The velocities given by Eq. 10 are compared with the velocities calculated by Thétis. As seen in Fig. 3, the velocity profiles are nearly identical. Near the beginning of the capillary rise, discrepancies of up to $5 \%$ may be found due to the fact that the initial condition in the numerical solution is zero velocity, but these differences are quickly reduced. Although the rest of the simulations in this work are 3D, the capillary pressure term does not require special treatment in extra dimensions, and the code is assumed to be applicable in $3 \mathrm{D}$.

\subsection{Gravity Tests}

The slurry parameter ranges are evaluated using a gravity test. The objective of this test is to explore acceptable parameter ranges, to find which parameters have a profound effect on results, and to gain insight into how the parameters affect impregnation results. The insight

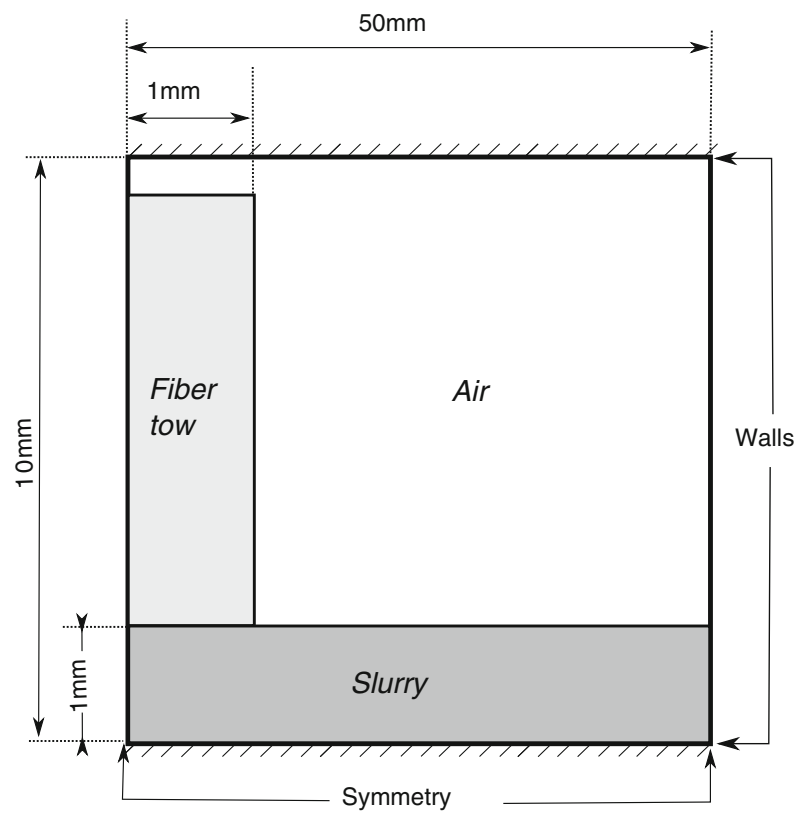

Fig. 2 2D fiber tow Thétis domain (not to scale) 

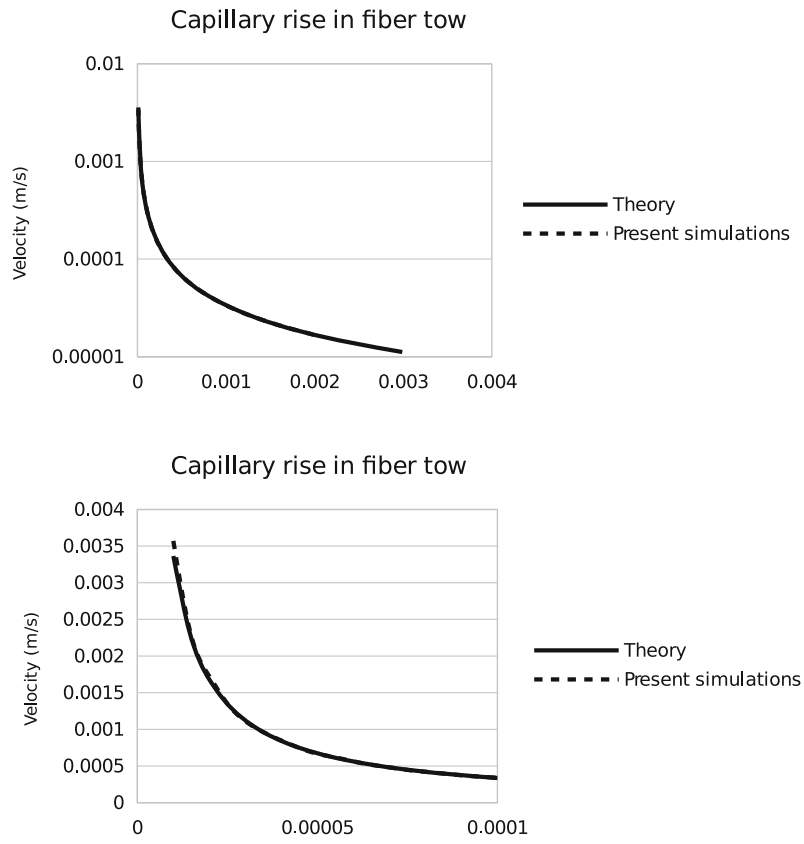

Fig. 3 Rise velocity comparison in a permeable fiber tow between present simulations and Darcy flow: (top) rise through first $2 \mathrm{~mm}$ of fiber tow, (bottom) zoom on first few moments of rise

gained here will allow further design of the slurry to improve impregnation. In this test, a rectangular mass of slurry is placed on the upper surface of one layer of the REV. The mass is placed so that its lower surface is just touching the fabric and so its center is centered on the fabric. The initial state is pictured in Fig. 4. The mass of slurry then descends into the fabric due to gravity and capillarity. In general, once the slurry has entered the fiber tows, the capillary pressure causes it to flow along the axial direction of the tows, transporting the slurry away from the center.

The effect of varying the slurry parameters is explored comparing with a baseline case (Case 0). The baseline case is designed with the slurry parameters of the currently used suspension. The parameters of each case have been defined in Table 1. The results pertaining to each test may be seen in Section 3.1.

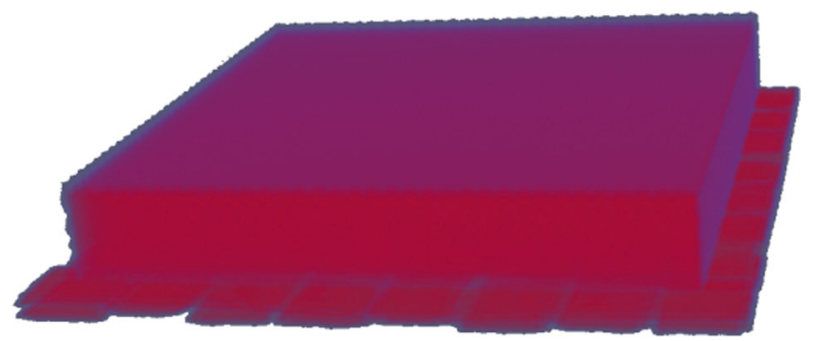

Fig. 4 Initial state for gravity tests 


\subsection{Bath Tests}

Once the parameter ranges are defined, the different processes may be evaluated for viability considering the slurry parameters. Whereas the gravity tests explore the slurry parameters, the process tests explore only the process parameters. The first process that is evaluated is open bath impregnation. In this process, the fabric is usually dragged through the open bath $[11,25]$. After being dragged out of the bath, the fabric can be routed through the bath another time in some cases. This pulling motion usually runs the fabric or single fiber tow through various rollers, which change the pulling direction. At the same time, these rollers will deform the fiber tow or fabric, affecting the macroporosity. Neither the rollers' influence on the fabric nor multiple submersions are taken into account in this study. The main parameter for this process is thus the velocity of pulling.

The numerical simulation places the fabric in the middle of the domain and imposes an inlet velocity in one of the tow directions. The opposing domain boundary is given Neumann ("free" outlet) conditions while the rest of the boundaries are given wall conditions. A schematic diagram of these conditions may be seen in Fig. 5.

\subsection{Wet Lay-up Tests}

The wet lay-up process spreads slurry on the top of the fabric at a set speed and angle. These tests approximate the addition of slurry with a dropper, for example, to the top surface of the fabric and the subsequent spreading of the slurry with a spatula. This type of process is normally referred to as hand lamination or wet lay-up [21]. The main parameters of this process that are modeled in this study are the angle and speed of advancement.

Numerically, this process involves an initial amount of slurry on top of the fabric. A set speed is imposed adjacent to the slurry, which along with the incline of the imposed velocity, applies pressure to the slurry. A schematic diagram of these conditions may be seen in Fig. 6.

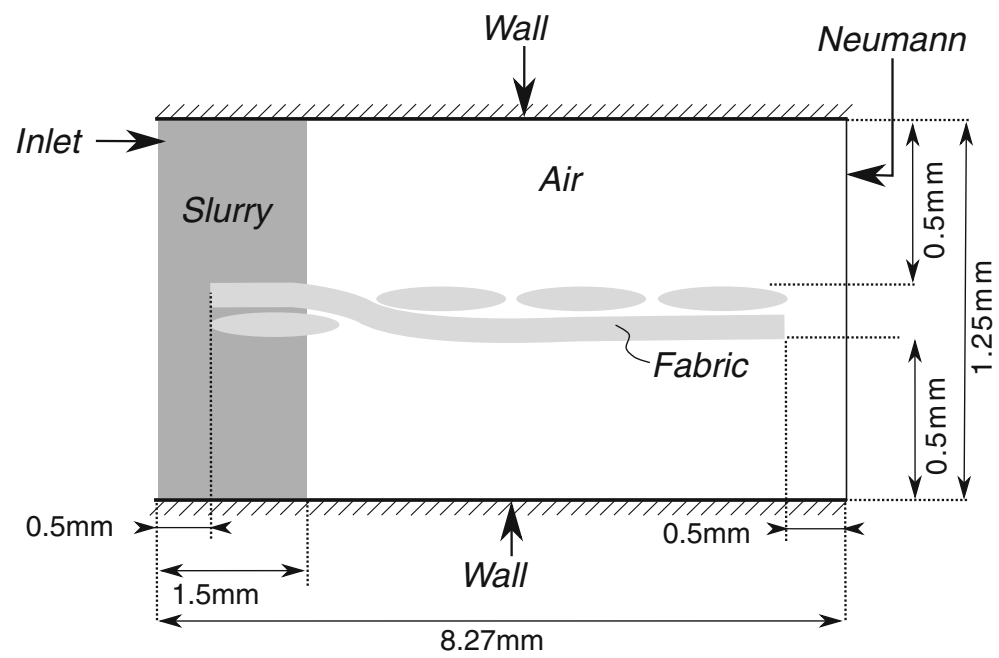

Fig. 5 Open bath impregnation test domain (not to scale): midplane parallel to flow \& perpendicular to fabric 


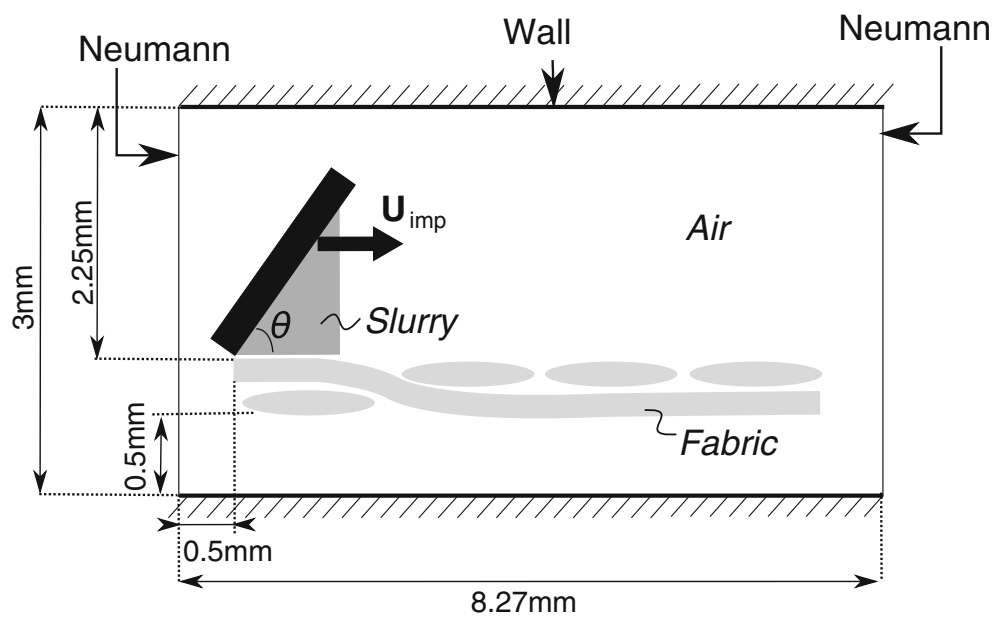

Fig. 6 Wet lay-up test domain (not to scale): midplane parallel to flow \& perpendicular to fabric

\section{Results}

\subsection{Gravity Test Results}

The results for the tests described in Section 2.5 may be seen in Table 3, with the parameters that are different from the baseline in bold. The results are compared using the saturation metric (see Eq. 11), where the tow saturation is calculated with respect to the total volume occupied by the fiber tows and the fabric saturation is calculated with respect to the total volume occupied by the fabric (including spacings in between the tows). The saturation equation is

$$
S=\frac{V_{l}}{V_{l}+V_{g}}
$$

Table 3 Tow saturation \& fabric saturation results after $16 \mathrm{~ms}$ for different parameter values in the gravity test. Bold text indicating the parameter value that has been varied with respect to Case 0

\begin{tabular}{lllllllll}
\hline & \multicolumn{2}{l}{ Input values } & & & & \multicolumn{2}{l}{ Model results } \\
\cline { 2 - 3 } Case & $\begin{array}{l}\text { Viscosity } \\
(\mathrm{Pa} \cdot \mathrm{s})\end{array}$ & $\begin{array}{l}\text { Density } \\
\left(\mathrm{kg} / \mathrm{m}^{3}\right)\end{array}$ & $\begin{array}{l}\text { Surface } \\
\text { tension }(\mathrm{N} / \mathrm{m})\end{array}$ & $\begin{array}{l}\text { Contact } \\
\text { angle }\left(^{\circ}\right)\end{array}$ & & $\begin{array}{l}\text { Tow } \\
\text { saturation }\end{array}$ & $\begin{array}{l}\text { Fabric } \\
\text { saturation }\end{array}$ \\
\hline 0 & 0.67 & 2092 & 0.047 & 60 & 0.485 & 0.701 \\
1 & $\mathbf{0 . 1 0}$ & 2092 & 0.047 & 60 & & 0.460 & 0.672 \\
2 & 0.67 & $\mathbf{2 5 4 0}$ & 0.047 & 60 & & 0.485 & 0.697 \\
3 & 0.67 & 2092 & $\mathbf{0 . 0 2}$ & 60 & & 0.486 & 0.695 \\
4 & 0.67 & 2092 & $\mathbf{0 . 0 8}$ & 60 & & 0.483 & 0.700 \\
5 & 0.67 & 2092 & 0.047 & $\mathbf{4 0}$ & & 0.484 & 0.693 \\
6 & 0.67 & 2092 & 0.047 & $\mathbf{8 0}$ & & 0.486 & 0.700 \\
\hline
\end{tabular}


where $S$ is the saturation, $V_{l}$ is the volume occupied by the liquid (the slurry), and $V_{g}$ is the volume occupied by the gas (air). The saturation evolution results within the fiber tows and the saturation evolution results within the fabric may be seen in Figs. 7 and 8.

According to these results, the most important slurry parameter is the viscosity. A lower viscosity helps flow within the fiber tows as well as outside the tows. During slurry design, the viscosity should thus be the primary concern.

Density variations on the other hand, which are influenced by the particle volume fraction and the solvent composition, have the smallest effect on the impregnation curves. The density was varied by $\approx 25 \%$ and gave no visible differences from the baseline results. It should also be kept in mind that the slurry density is homogeneous throughout the simulation domain and that density variations resulting from volume fraction variations could possibly change the saturation evolution curves. Density variations also usually cause viscosity variations, but this is not studied in this article.

The remaining slurry parameters, surface tension and contact angle, have similar effects on the saturation evolution within fiber tows. The parameter range limits set forth in this study for these two parameters are somewhat arbitrary but are representative of the feasible variations during slurry fabrication. The saturation results for the limits of the parameter ranges are nearly identical, suggesting that these two parameters are approximately equal in importance when designing a slurry to impregnate the fiber tows. This is not surprising considering that the highest surface tension $(0.08 \mathrm{~N} / \mathrm{m})$ and the lowest contact angle $\left(40^{\circ}\right)$ result in similar capillary pressures of $15322 \mathrm{~Pa}$ and $13790 \mathrm{~Pa}$ respectively, and the lowest surface tension $(0.02 \mathrm{~N} / \mathrm{m})$ and the highest contact angle $\left(80^{\circ}\right)$ result in similar capillary pressures of $3831 \mathrm{~Pa}$ and $3126 \mathrm{~Pa}$ respectively.

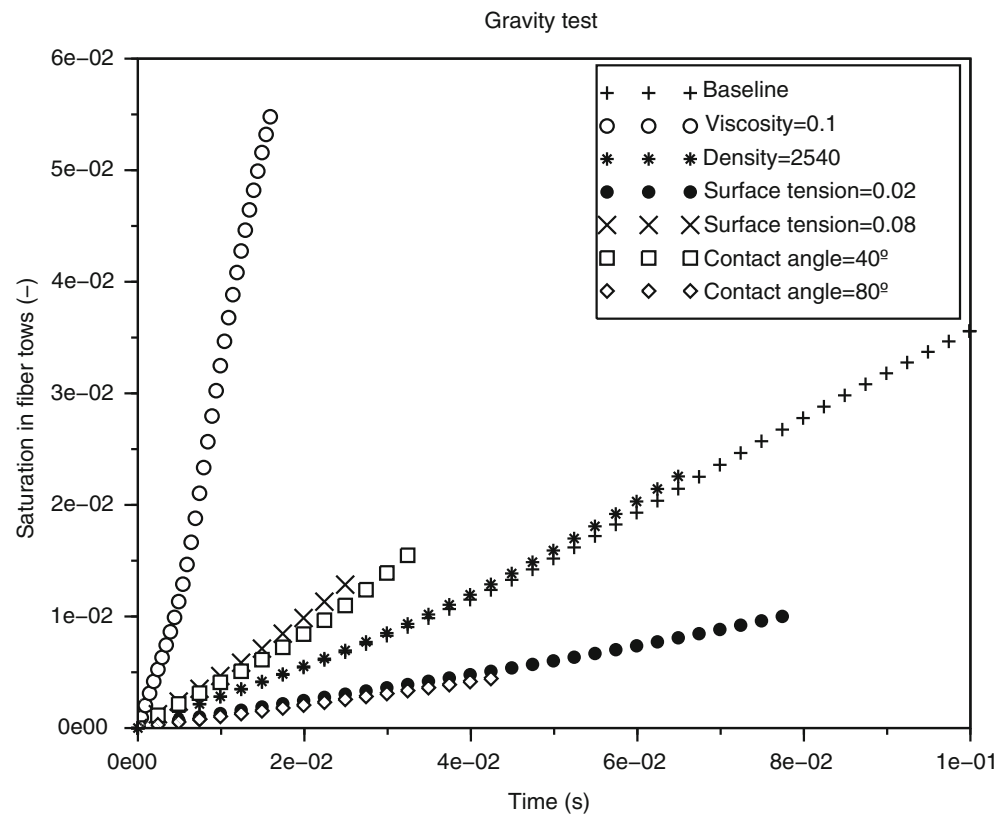

Fig. 7 Saturation time evolution within fiber tows for different slurry parameter sets. Baseline: $\mu=0.67 P a$. $s, \rho=2092 \mathrm{~kg} / \mathrm{m}^{3}, \sigma=0.047 \mathrm{~N} / \mathrm{m}, \theta=60^{\circ}$; other cases vary only 1 parameter from baseline 


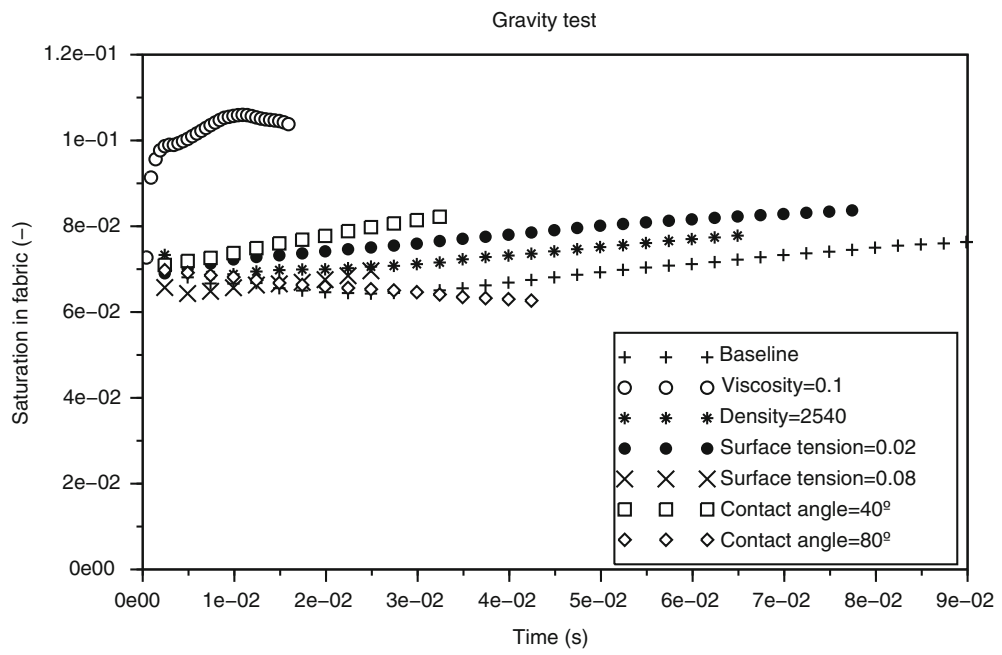

Fig. 8 Saturation time evolution within entire fabric (bottom) for different slurry parameter sets. Baseline: $\mu=0.67 \mathrm{~Pa} \cdot \mathrm{s}, \rho=2092 \mathrm{~kg} / \mathrm{m}^{3}, \sigma=0.047 \mathrm{~N} / \mathrm{m}, \theta=60^{\circ}$; other cases vary only 1 parameter from baseline

The surface tension also affects the slurry flow between fiber tows, and a higher surface tension causes a less complete impregnation of the fabric (see the " $x$ " data set in Fig. 8). This suggests that less air bubbles will be trapped in the fiber tows if the flow is relatively faster within the fiber tows. It should be noted once again that the wetting effects outside the fiber tows are assumed to be negligible. A more complete study, taking into account the wetting effects outside the tows could cause these results to shift, but it is assumed that this shift will be insignificant.

\subsection{Bath Tests}

The results for the tests described in Section 2.6 may be seen in Table 4. The saturation evolution results within the fiber tows and within the fabric may be seen in Figs. 9 and 10 .

As can clearly be seen in Figs. 9 and 10, the saturation within and outside of the fiber tows evolves more or less at the same rate for 0.0001 and $0.001 \mathrm{~m} / \mathrm{s}$ inlet velocities. In fact, at these impregnation speeds, the capillary forces seem to be dominant, with most of the impregnation within the tows occurring due to the capillary forces. The $0.003 \mathrm{~m} / \mathrm{s}$ inlet velocity starts to break away from the results for smaller velocities. Although on short time scales these results seem to indicate a better impregnation for higher velocities, the

Table 4 Tow saturation \& fabric saturation results after $27.8 \mathrm{~ms}$ for different inlet velocities in the bath test

\begin{tabular}{llll}
\hline Case & Inlet velocity $(\mathrm{m} / \mathrm{s})$ & Tow saturation & Fabric saturation \\
\hline 7 & 0.0001 & 0.41983 & 0.6235 \\
8 & 0.0010 & 0.41981 & 0.6230 \\
9 & 0.0030 & 0.41974 & 0.6201 \\
10 & 0.0070 & 0.41960 & 0.6137 \\
11 & 0.0100 & 0.41949 & 0.6081 \\
\hline
\end{tabular}




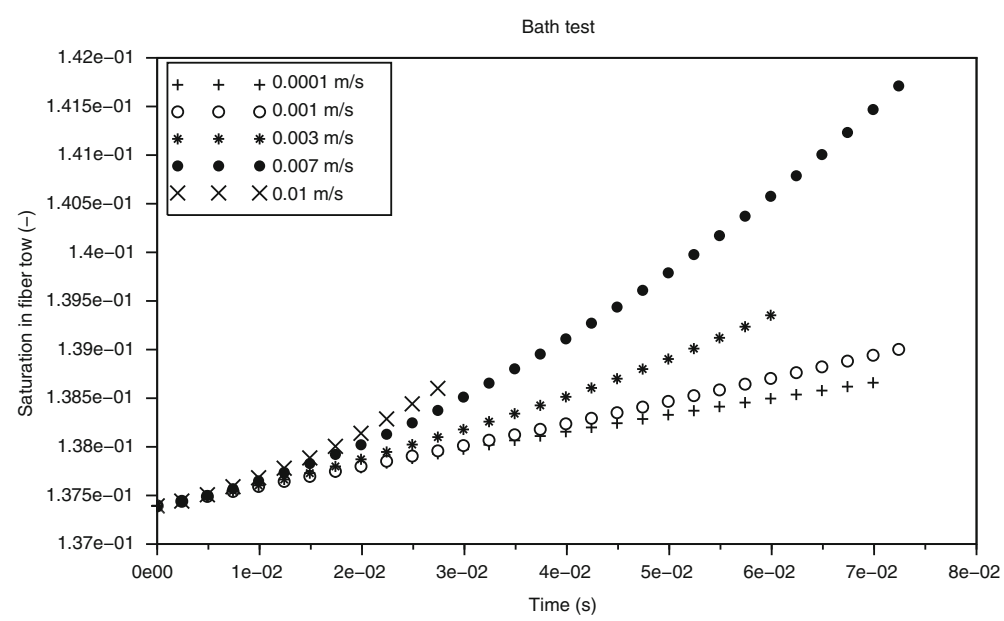

Fig. 9 Saturation time evolution within fiber tows for different open bath impregnation speeds

implications are quite different. Since the saturation within the fabric at $0.003 \mathrm{~m} / \mathrm{s}$ evolves at a much faster pace than for smaller velocities, there is a very real possibility that the flow around fiber tows is too fast, causing air to become trapped within fiber tows. Unfortunately, due to prohibitive simulation times, this hypothesis cannot be confirmed, but it is supported by inspection of the saturation profiles.

The beginnings of air entrapment may be seen in the saturation profiles, with a zoom of the area surrounding the initial slurry front shown in Fig. 11 for the initial condition, inlet velocity $=0.0001 \mathrm{~m} / \mathrm{s}$, inlet velocity $=0.001 \mathrm{~m} / \mathrm{s}$, and inlet velocity $=0.1 \mathrm{~m} / \mathrm{s}$ respectively. In these profiles, gray represents the slurry, and the fiber tows are outlined. In particular, the cross-section of a tow perpendicular to the inlet velocity may be seen on bottom while a

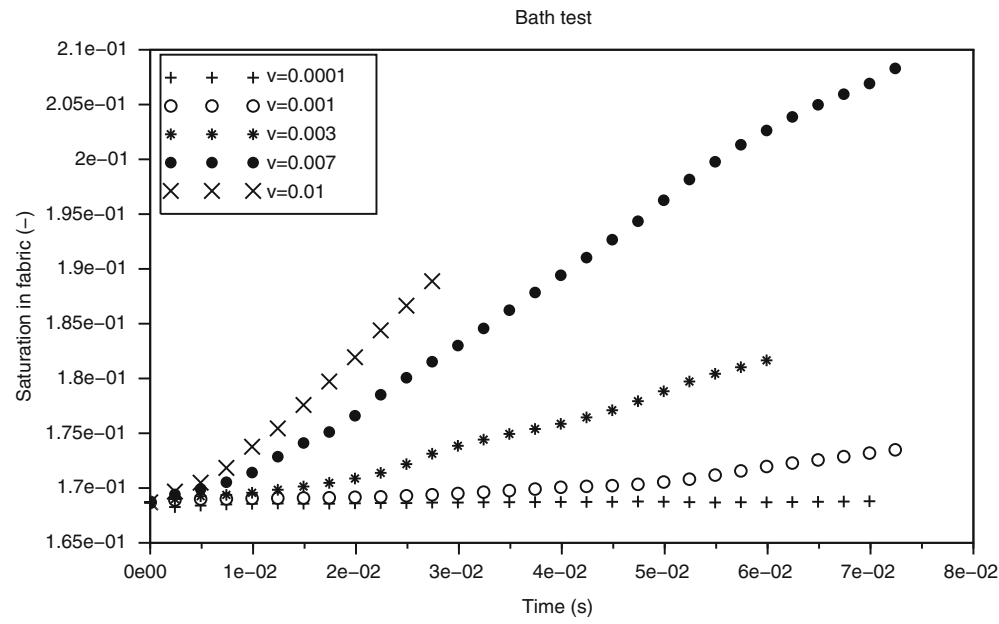

Fig. 10 Saturation time evolution within entire fabric for different open bath impregnation speeds 


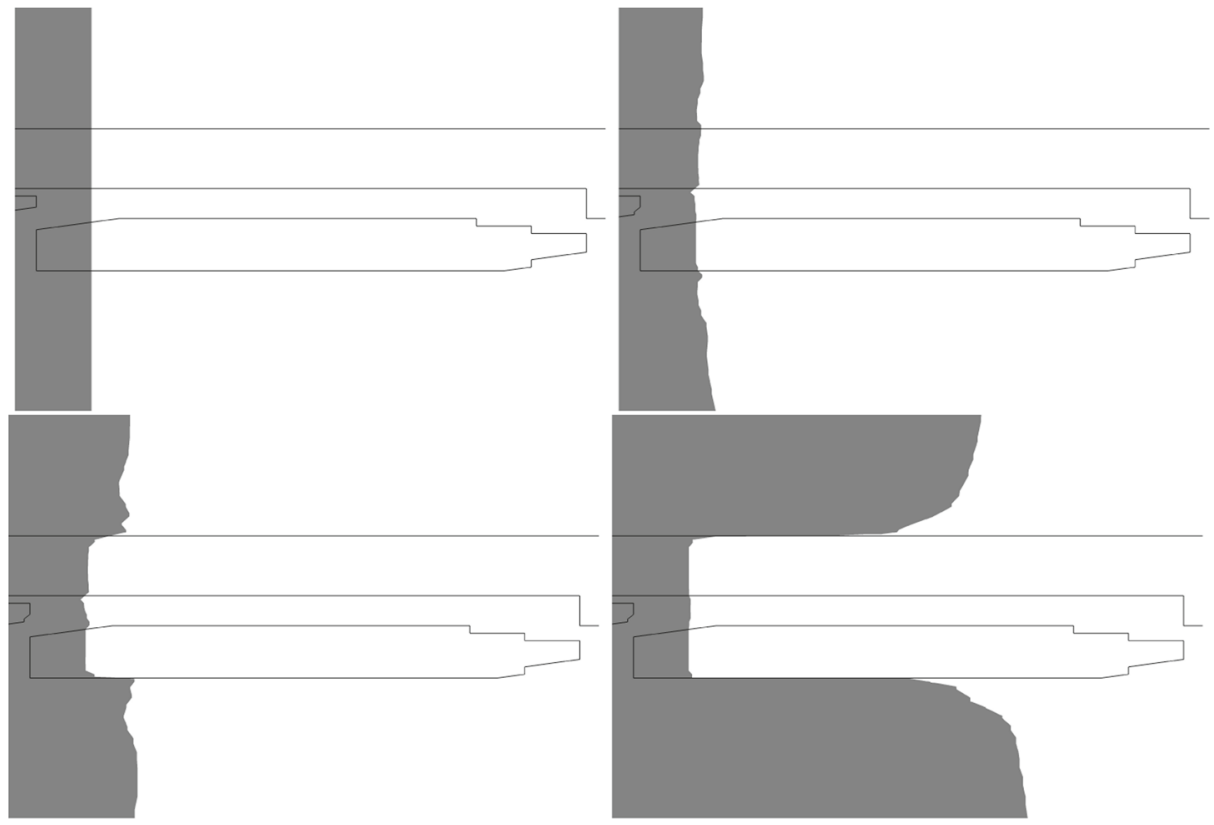

Fig. 11 Saturation profile (top left) at the beginning of the simulation $\&$ in the first few moments for inlet velocities of (top right) $0.0001 \mathrm{~m} / \mathrm{s}$, (bottom left) $0.001 \mathrm{~m} / \mathrm{s}$, and (bottom right) $0.1 \mathrm{~m} / \mathrm{s}$

tow parallel to the inlet velocity may be seen on top. In the top-right profile of Fig. 11, the capillary flow within the fiber tows in the axial direction is of the same order as the inlet velocity $(0.0001 \mathrm{~m} / \mathrm{s})$. This can be seen in the fiber tow outlined on top, where the slurry advances more or less at the same rate as the flow far away from the fabric. The flow in the perpendicular direction (or into and through the bottom tow) is slower than the inlet velocity due to the smaller permeability and capillary pressure in the perpendicular direction. At a higher velocity, the flow outside the fabric is already faster than the capillary speed within the tows. The saturation profiles show that at $0.001 \mathrm{~m} / \mathrm{s}$ (see bottom-left in Fig.11), the inlet velocity is larger than the capillary speed although it is unclear whether air will become trapped within the fiber tows. At much higher speeds, such as $0.1 \mathrm{~m} / \mathrm{s}$ (see bottom-right in Fig. 11), it is clear that air will become trapped within the tows. It would be interesting to confirm whether winding the fabric through various rollers would help expel these air pockets, this could be investigated in a future study.

\subsection{Wet Lay-up Tests}

The results for the tests described in Section 2.7 may be seen in Table 5. The saturation evolution results within the fiber tows and the saturation evolution results within the fabric may be seen in Figs. 12 and 13. It should be noted that none of the simulations were carried out until full saturation. Unfortunately, the simulation times were too long, and in the future, a more complete saturation could be studied with more computational resources.

Higher spreading speeds seem to improve impregnation, but this would need to be confirmed after full impregnation of the fabric. There is a bigger difference between $0.01 \mathrm{~m} / \mathrm{s}$ 
Table 5 Tow saturation \& fabric saturation results after $31.2 \mathrm{~ms}$ for different lay-up velocities \& angles in the wet lay-up test

\begin{tabular}{lllll}
\hline Case & Inlet velocity $(\mathrm{m} / \mathrm{s})$ & Angle $\left({ }^{\circ}\right)$ & Tow saturation & Fabric saturation \\
\hline 13 & 0.0001 & 90 & $7.69 \cdot 10^{-} 4$ & $1.01 \cdot 10^{-2}$ \\
14 & 0.0010 & 90 & $7.45 \cdot 10^{-} 4$ & $9.58 \cdot 10^{-3}$ \\
15 & 0.0100 & 90 & $6.47 \cdot 10^{-} 4$ & $7.54 \cdot 10^{-3}$ \\
17 & 0.0010 & 45 & $1.96 \cdot 10^{-} 3$ & $2.13 \cdot 10^{-} 2$ \\
\hline
\end{tabular}

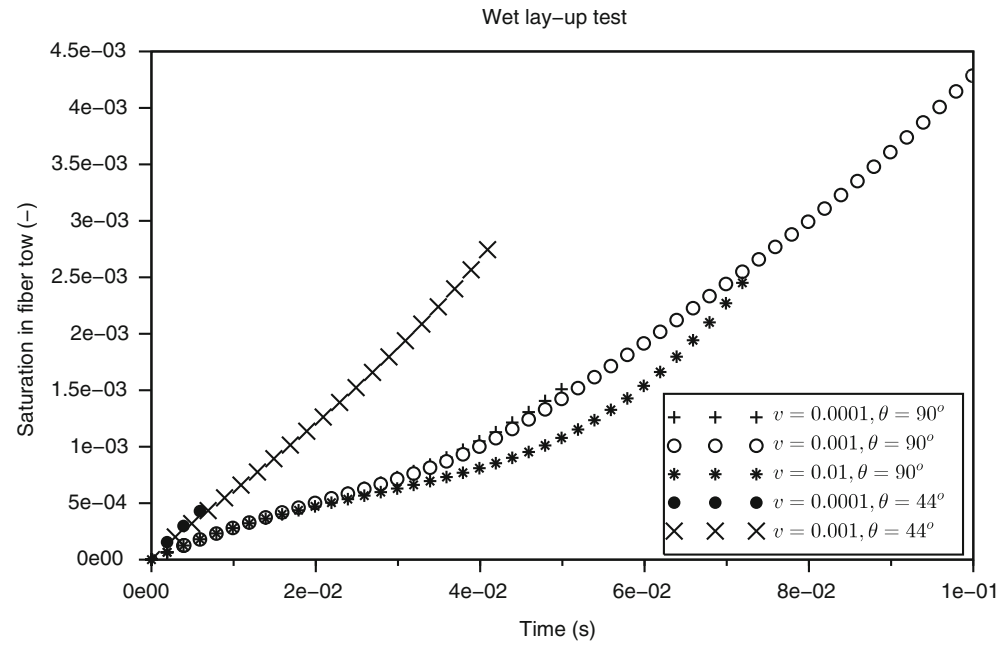

Fig. 12 Saturation time evolution within fiber tows for angles and spreading speeds for wet lay-up

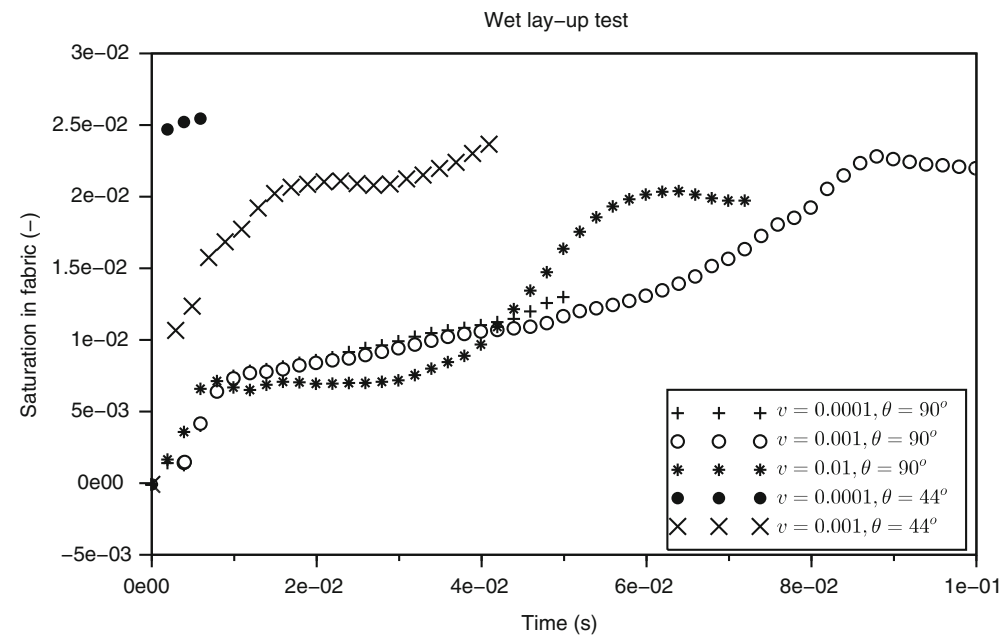

Fig. 13 Saturation time evolution within entire fabric for angles and spreading speeds for wet lay-up 
and $0.001 \mathrm{~m} / \mathrm{s}$ than $0.001 \mathrm{~m} / \mathrm{s}$ and $0.0001 \mathrm{~m} / \mathrm{s}$. This indicates that the final results, which will take into account trapped air bubbles, would be different for larger velocities. Nevertheless, further investigations, with longer simulation time, must be done to confirm this hypothesis.

As expected, an inclined spreading angle gives better impregnation within the fabric as well as within the tows due to the increased pressure forcing the slurry into and around the tows. Unexpectedly, however, the slower spreading speeds give better impregnation of the fabric at slower speeds. This is most likely due to the slurry having more time to impregnate the fabric between the tows before being pushed to intertows further along. Notwithstanding, adding enough slurry will most likely lead to perfect saturation between fiber tows. The most important parameter will thus be the incline angle as this will affect the tow impregnation.

\section{Conclusions}

This study focuses on the effect of slurry and process parameters on impregnation of a specific ceramic fabric. Impregnation for this particular fabric and slurry is relatively uncharted terrain, and thus requires extensive testing to achieve the best final product mechanical properties. It is assumed in this context that the most complete impregnation of the fabric will give the best mechanical properties. For this reason, this study focuses on achieving the most complete infiltration possible, with a macroporosity of 0 .

Viscosity, surface tension, density, and contact angle were considered to be the most significant of the slurry parameters. Ranges were set for each of these parameters based on realistically obtainable values, which depend on particle concentration and solvent content. Slurries corresponding to each of the range limits were then tested, allowing the impact of each parameter to be contrasted with the baseline configuration. In this way, the slurry viscosity was shown to be the most important parameter for the impregnation saturation time evolution. Density, on the other hand, was the least important of the slurry parameters. The surface tension and contact angle feasible limits gave similar results within the fiber tows. The surface tension results for within and outside fiber tows were however contrasting since a higher surface tension gave faster impregnation within the fiber tows but slower impregnation of the fabric. Nonetheless, this contrasting behavior will more likely ensure that the fiber tows are filled before being completely enveloped by the slurry and having air bubbles trapped within them.

Besides the slurry parameters that were studied, two different processes and their corresponding parameters were studied. In particular, the impregnation speed of open bath impregnation and the spreading speed and angle for wet lay-up were studied. The open bath impregnation tests showed that for impregnation speeds of up to $0.001 \mathrm{~m} / \mathrm{s}$ the impregnation was mostly piloted by capillary effects. At higher impregnation speeds, the saturation evolution time evolution plots had a much larger dependence on the impregnation speed, implying that there would possibly be air bubbles trapped in the fiber tows. This effect can be seen in the saturation profiles within the fabric. Complete simulations for much longer simulation times would help corroborate this assumption, but due to prohibitive simulation times, this was not possible. It is clear however that at most pulling speeds, air bubbles will become trapped within the fabric. It is probably necessary to apply an extra step in order to minimize these air bubbles, such as immersing multiple times in the bath, applying pressure with rollers, etc. 
The second process that was studied was the wet lay-up process. Different angles and speeds were studied for this process. Higher spreading speeds slightly improve impregnation, but the differences may be smaller for full impregnation. The most important parameter here is the incline angle for the spreading process. The incline applies pressure to the slurry, allowing it to overcome the Darcy head loss easier. Further studies of the incline angle and speeds would be necessary to prepare the ideal wet lay-up process.

In future studies, it would be interesting to add wetting effects outside the fiber tows to completely evaluate their importance. At the same time, the segregation of the slurry remains to be determined and whether it has a large effect on the impregnation results.

Acknowledgements The results presented here were obtained as part of the research project "CMC oxydes voie liquide" at IRT Saint Exupéry in collaboration with I2M. We would like to the thank the industrial and academic partners who supported this project financially. We would also like to thank the Commissariat Général aux Investissements et l'Agence Nationale de la Recherche (France) for their financial support as part of the Programme d'Investissement d'Avenir (PIA). This project, carried out in Bordeaux, received indirect aids from the Aquitaine region, and we wish to thank them.

The authors wish to thank Aude Paillassa, Alexandre Lahutte, Florent Deliane, Natacha Duée, and Rémi Bouvier for their help with fabricating the slurries and conducting the tests to determine the slurry parameters. The authors would also like to thank Stéphane Glockner for his help with numerical methods and model initial and boundary conditions, and Nicolas Guel for his help in creating the fabric geometry.

Computer time for this study was provided by the computing facilities MCIA (Mésocentre de Calcul Intensif Aquitain) of the Université de Bordeaux and of the Université de Pau et des Pays de l'Adour. This work was granted access to the HPC resources of CINES under the allocation x20162b7660 made by GENCI (Grand Equipement National de Calcul Intensif).

Open Access This article is distributed under the terms of the Creative Commons Attribution 4.0 International License (http://creativecommons.org/licenses/by/4.0/), which permits unrestricted use, distribution, and reproduction in any medium, provided you give appropriate credit to the original author(s) and the source, provide a link to the Creative Commons license, and indicate if changes were made.

\section{References}

1. Advani, S., Dimitrovova, Z.: Chapter 8 - role of capillary flow in composite manufacturing. In: Hartland, S. (ed.) Surface and Interfacial Tension: Measurement, theory, and applications, volume 119 of Surfactant Science Series. CRC Press (2004)

2. Ahn, K.J., Seferis, J.C., Berg, J.C.: Simultaneous measurements of permeability and capillary pressure of thermosetting matrices in woven fabric reinforcements. Polym. Compos. 12(3), 146-152, 6 (1991)

3. Amico, S., Lekakou, C.: Mathematical modelling of capillary micro-flow through woven fabrics. Compos. A: Appl. Sci. Manuf. 31(12), 1331-1344 (2000)

4. Amico, S., Lekakou, C.: An experimental study of the permeability and capillary pressure in resintransfer moulding. Compos. Sci. Technol. 61(13), 1945-1959 (2001)

5. Arquis, E., Pons, A., Dugois, K., Lebot, C., Valat, M., Lasseux, D., Vincent, S., Descamps, C.: Ecoulements capillaires et chargés dans les procédés d'élaboration de composites par APS et RMI. In: 22ème Congrès Français de Mécanique, number 71273 (2015)

6. Baché, E., Le Bot, C., Fereres, S., Palomo, E.: Off-eutectic binary salt finite volume method. Energy Procedia 49(0), 715-724 (2014). Proceedings of the SolarPACES 2013 International Conference

7. Benazzouk, L., Arquis, E., Bertrand, N., Descamps, C.: Numerical calculations of dynamic behavior of a rotating ceramic composite with a self-healing fluid. Ceram. Eng. Sci. Proc. 34(10), 173-184 (2014)

8. Brackbill, J.U., Kothe, D.B., Zemach, C.: A continuum method for modeling surface tension. J. Comput. Phys. 100(2), 335-354 (1992)

9. Brinkman, H.C.: A calculation of the viscous force exerted by a flowing fluid on a dense swarm of particles. Appl. Sci. Res. A1, 27-34 (1947)

10. Bruneau, C.-H., Mortazavi, I.: Controle passif d'ecoulements incompressibles autour d'obstacles a l'aide de milieux poreux. Comptes Rendus de l'Academie des Sciences Series IIB Mechanics 329(7), 517-521 (2001) 
11. Campbell, F.C.: Structural composite materials. ASM International (2010)

12. Carman, P.C.: Fluid flow through granular beds. Chem. Eng. Res. Design, 75 (1997)

13. Danis, M., Del Borrello, C., Lacoste, E., Mantaux, O.: Infiltration of fibrous preform by a liquid metal Modelization of the preform deformation. In: Proceeding of the ICCM, p. 12 (1999)

14. Darcy, H.: Les fontaines publiques de la ville de Dijon. Victor Dalmont, Paris (1856)

15. de Oliveira, I., Amico, S., Souza, J., de Lima, A.: Resin transfer molding process: a numerical and experimental investigation. Int. J. Multiphys. 7(2), 125-136 (2013)

16. Desombre, J., Morichon, D., Mory, M.: Simultaneous surface and subsurface air and water flows modelling in the swash zone. Coast. Eng. Proc. 1(33), 56 (2012)

17. Devalve, R.C., Pitchumani: Numerical simulation of air entrapment during resin transfer modeling. In: SAMPE 2011. Long Beach (2011)

18. Gebart, B.R.: Permeability of unidirectional reinforcements for rtm. J. Compos. Mater. 26(8), 1100-1133 (1992)

19. Guillaument, R., Vincent, S., Caltagirone, J.P.: An original algorithm for vof based method to handle wetting effect in multiphase flow simulation. Mech. Res. Commun. 63(Complete), 26-32 (2015)

20. Halbig, M.C., Jaskowiak, M.H., Kiser, J.D., Zhu, D.: Evaluation of ceramic matrix composite technology for aircraft turbine engine applications. In: 51st AIAA Aerospace Sciences Meeting Including the New Horizons Forum and Aerospace Exposition, pp. 07-10 (2013)

21. Hoa, S.V.: Principles of the Manufacturing of Composite Materials. DEStech Publications, Incorporated (2009)

22. Hotza, D., Guglielmi, P.O., Nunes, G.F., Hablitzel, M., Janssen, R.: Production of oxide ceramic matrix composites by a prepreg technique. In: Advanced Powder Technology VIII, volume 727 of Materials Science Forum, pp. 556-561. Trans Tech Publications 11 (2012)

23. Hu, J.: 8 - permeability of multilayer woven fabrics. In: Hu, J. (ed.) 3-D Fibrous Assemblies, Woodhead Publishing Series in Textiles, pp. 194-220. Woodhead Publishing (2008)

24. Krenkel, W., Dollhopf, V.: Rtm processing of high performance composites. In: Proceedings of ICCM2011. Gold Coast Australia (1997)

25. Miaris, M., Paessler, A., Schledjewski, R., Mitschang, P.: Modeling the impregnation process of a siphon impregnation system during filament winding. In: ASME Pressure Vessels and Piping Conference. Baltimore (2011)

26. Mileiko, S.T.: Chapter $\{\mathrm{XIII}\}$ - liquid infiltration. In: Mileiko, S.T. (ed.) Metal and Ceramic Based Composites, volume 12 of Composite Materials Series, pp. 547-586. Elsevier (1997)

27. Nabovati, A., Llewellin, E.W., Sousa, A.CM.: A general model for the permeability of fibrous porous media based on fluid flow simulations using the lattice boltzmann method. Compos. A: Appl. Sci. Manuf. 40(6), 860-869 (2009)

28. Nowak, J.H., Chun, T., Berdichevsky, A.L., Cai, Z.: Flow visualization of fiber impregnation in resin transfer molding. In: 1st International Conference on Transport Phenomena in Food Processing. Honolulu (1992)

29. Rady, M., Arquis, E., Gobin, D., Goyeau, B.: Numerical simulation of channel segregates during alloy solidification using tvd schemes. Int. J. Numer. Method. H. 20, 841-866 (2010)

30. Rady, M., Arquis, E.: A dual-scale coupled micro/macro segregation model for dendritic alloy solidification. Heat Mass Transf. 42(12), 1129-1141 (2006)

31. Senoguz, M.T., Dungan, F.D., Sastry, A.M., Klamo, J.T.: Simulations and experiments on low-pressure permeation of fabrics: Part II-the variable gap model and prediction of permeability. J. Compos. Mater. 35, 1285-1322 (2001)

32. Thétis: Code de mécanique des fluides. http://thetis.enscbp.fr/. Accessed 30 June 2014 